\title{
Irreducible Apollonian Configurations and Packings
}

\author{
Steve Butler • Ron Graham • Gerhard Guettler • \\ Colin Mallows
}

Received: 18 January 2009 / Revised: 20 July 2009 / Accepted: 20 July 2009 /

Published online: 1 August 2009

(C) The Author(s) 2009. This article is published with open access at Springerlink.com

\begin{abstract}
An Apollonian configuration of circles is a collection of circles in the plane with disjoint interiors such that the complement of the interiors of the circles consists of curvilinear triangles. One well-studied method of forming an Apollonian configuration is to start with three mutually tangent circles and fill a curvilinear triangle with a new circle, then repeat with each newly created curvilinear triangle. More generally, we can start with three mutually tangent circles and a rule (or rules) for how to fill a curvilinear triangle with circles.

In this paper we consider the basic building blocks of these rules, irreducible Apollonian configurations. Our main result is to show how to find a small field that can realize such a configuration and also give a method to relate the bends of the new circles to the bends of the circles forming the curvilinear triangle.
\end{abstract}

Keywords Irreducible · Apollonian · Packing · Eulerian · Inversion

S. Butler supported by an NSF Postdoctoral fellowship.

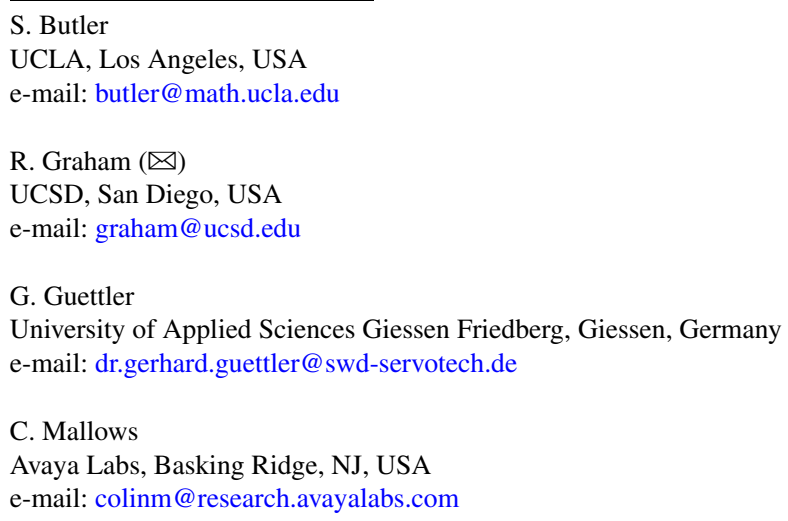




\section{Introduction}

An Apollonian configuration of circles is a collection of circles in the plane with disjoint interiors such that the complement of the interiors of the circles consists of curvilinear triangles. Such configurations have been studied before as special cases of circle packing (see $[11,12]$ ). In examining these configurations it is often more convenient to consider the bend of the circle (one over the radius) than the radius itself.

Perhaps the most well-known, and most studied, example of an Apollonian configuration is formed by starting with three mutually tangent circles and then filling in each curvilinear triangle with the unique circle which is tangent to all three sides of that triangle (see Fig. 1a); we then repeat this process with each newly created curvilinear triangle as often as desired. This has the remarkable property that if the first three circles have integer bends $a, b, c$ and

$$
\langle a, b, c\rangle:=a b+a c+b c
$$

is also the square of an integer, then each new circle which is added will also have integer bend. Further, for any three mutually tangent circles with bends $d, e, f$ then $\langle d, e, f\rangle=m^{2}$ for $m$ an integer. These are consequences of Descartes Circle Theorem. The properties of this configuration have been extensively studied (see [4-7]).

However, there are other ways to fill in a curvilinear triangle. Recently Guettler and Mallows [8] examined the case where the curvilinear triangle is filled by three new circles, each tangent to exactly two sides (see Fig. 1b). This also has a similar property in that if the first three circles have integer bends $a, b, c$ and $\langle a, b, c\rangle=$ $2 m^{2}$ for $m$ an integer, then each new circle will also have integer bend. Further, for any three mutually tangent circles with bends $d, e, f$ then $\langle d, e, f\rangle=2 m^{2}$ for $m$ an integer. (This additional factor of 2 plays an important role in the packing, as we will see in Sect. 3.)

In both of these cases the important element of the packing is the recursive rule for filling in the curvilinear triangles. The basic building blocks for forming these rules are the irreducible Apollonian configurations which we will introduce in Sect. 2. In

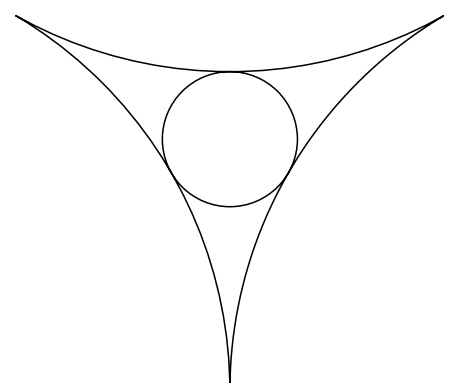

(a) Standard Apollonian rule.

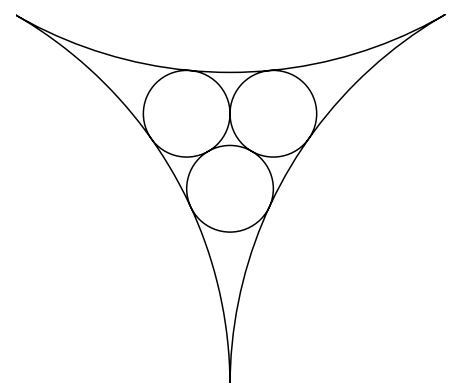

(b) Guettler-Mallows rule.

Fig. 1 Two rules for packing a curvilinear triangle 
Sect. 3 we will look at the problem of determining a small field that can be used to represent a configuration (irreducible or not). In Sect. 4 we will show how to take an Apollonian configuration and construct a rule for filling a curvilinear triangle. In Sect. 5 we give some concluding remarks.

\section{Irreducible Apollonian Configurations}

There are several ways to represent an Apollonian configuration. Combinatorially it can be represented as a tangency graph where each circle is a vertex and tangent circles are joined by an edge. The resulting graph is a planar triangulated graph, which corresponds to a triangulation of the sphere.

Theorem 1 (Koebe-Andreev-Thurston [11]) Given a triangulation of the sphere, there exists an essentially unique circle packing where circles correspond to vertices and edges to tangency between circles. Moreover, by projection this can be realized as a circle packing in the plane, and any two circle packings in the plane corresponding to the triangulated graph differ by a Moebius transformation.

In Fig. 2a we give a planar triangulated graph. One circle packing in the plane that realizes this configuration is shown in Fig. $2 \mathrm{~b}$ (the outer circle has negative bend, so its interior lies on the outside of the disc). There are of course many possible ways to realize the configuration by transforming the packing using a Moebius transformation.

We will see that when looking for a small field that can be used to represent the packing, an important type of packing is one where we have a unit circle centered at $(0,0)$ and two circles with bend 0 located at $y=1$ and $y=-1$. We will call such a packing a standard packing. One standard packing for Fig. 2a is shown in Fig. 2c.

Every packing can be transformed into a standard packing by inverting at a circle centered at a point of tangency, then rotating, scaling, and translating to put it into the correct position. In general, standard packings are not unique, since by choosing to invert at a different point of tangency we will be led to a (possibly) different standard packing. However, since there are only finitely many points of tangency, there are only finitely many standard packings. By using $V-E+F=2$ we have the following.

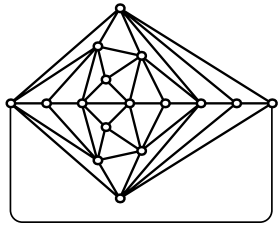

(a) Tangency graph

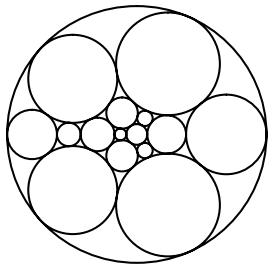

(b) Generic packing

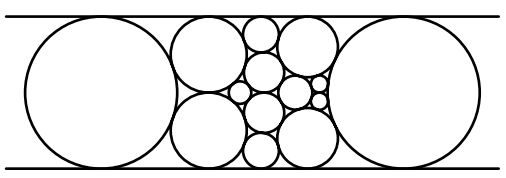

(c) Standard packing

Fig. 2 Different representations of an Apollonian packing 


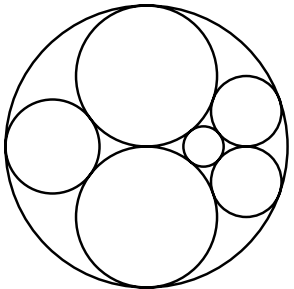

(a) configuration (not irreducible)

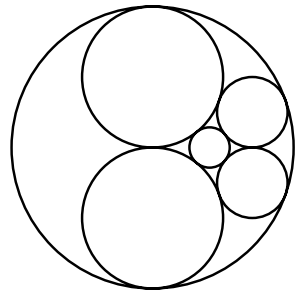

(b) configuration (irreducible)

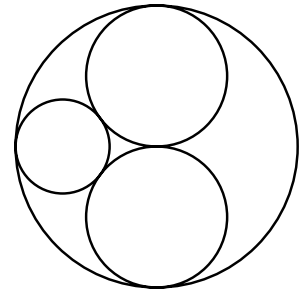

(c) configuration (irreducible)

Fig. 3 Example of decomposing a configuration into irreducible parts

Lemma 1 Let $G$ be a planar triangulated graph with $n$ vertices (so that an associated packing will have $n$ circles). Then there are at most $3 n-6$ different standard packings with tangency graph $G$.

In this paper we will focus on irreducible Apollonian configurations. In terms of the tangency graph, this corresponds to having no triangles that are not faces. In terms of a packing, this is equivalent to saying that no proper subset of circles is also a nontrivial Apollonian configuration (trivial means three mutually tangent circles).

Starting with a tangency graph, if we have a triangle which is not a face, we can decompose the graph into two parts: the triangle with the interior vertices and edges; and the triangle with the exterior vertices and edges. We can continue doing this until each graph is irreducible, or in other words, we can decompose the tangency graph into irreducible components which are glued together on triangular faces. We can do the analogous procedure for the packing in that we can break it into irreducible packings that are glued together on three circles. An example of this is shown in Fig. 3, where we have a packing which is not irreducible and then show the two irreducible components in the packing.

So when we want to study properties of Apollonian packings, we can focus on the building blocks which are the irreducible components of the packing. There are many such irreducible Apollonian configuration with $n$ circles. Starting with $n=4$, there are $(1,0,1,1,2,4,10,25,87,313,1357,6244,30926,158428, \ldots)$ such configurations (see A007021 in [10], which differs in the $n=5$ case; also see [1]).

\section{Finding a Small Field for an Apollonian Configuration}

We now consider the problem of finding a small (ideally smallest) field $\mathbb{F}$ that can be used to represent an Apollonian packing. Here to represent a packing we mean that the bends and the centers of the circles can be expressed using elements of the field $\mathbb{F}$, as described below.

If we compare the two different packings mentioned in the introduction, we see that one of them satisfies $\langle a, b, c\rangle=m^{2}$, while the other satisfies $\langle a, b, c\rangle=2 m^{2}$. This factor of 2 in the second case plays an important role in the packing. In general we will say that a packing over a field $\mathbb{F}$ is a $q$-packing, for some fixed $q \in \mathbb{F}$, if the 
bends of all the circles are in $\mathbb{F}$ and further any three mutually tangent circles with bends $a, b, c$ satisfy $\langle a, b, c\rangle=q m^{2}$ for some $m$ in $\mathbb{F}$. Note that for every packing, by enlarging the field (i.e., $\mathbb{F}=\mathbb{R}$ ) we can ensure that the packing is a 1-packing. The interesting cases are where for some field, $q$ is not a square. Examples are given in some of the figures below where $q$ is not a square.

In our packing we can represent every circle by the triple $(\sqrt{q} x, y ; b)$ where $(\sqrt{q} x, y)$ is the center and $b$ is the bend. The tangency relationship between two circles with nonzero bend translates into the equation

$$
q\left(x_{1}-x_{2}\right)^{2}+\left(y_{1}-y_{2}\right)^{2}=\left(\frac{1}{b_{1}}+\frac{1}{b_{2}}\right)^{2} .
$$

A circle with bend 0 (which corresponds to a straight line in the diagram) would be described by $(\infty, \infty ; 0)$. This does not uniquely describe the line. So in this case we will represent the circle by the line $y=\sqrt{q} m x+b$ or $x=\sqrt{q} a$; equivalently we have that the line passes through two points of the form $\left(\sqrt{q} x_{1}, y_{1}\right)$ and $\left(\sqrt{q} x_{2}, y_{2}\right)$. (For most of this paper, we will see that we can assume that it is of the form $y=b$.) The tangency relationship between a circle $\left(\sqrt{q} x_{0}, y_{0} ; b_{0}\right)$ and the circle $y=\sqrt{q} m x+b$ then becomes

$$
\frac{q m^{2}+1}{b_{0}^{2}}=\left(y_{0}-q m x_{0}-b\right)^{2} .
$$

This gives a system of polynomial equations that has a solution in some algebraic number field. (The solution is of course far from unique; in practice we first "anchor" three circles by choosing centers and bends for them and then solve for the remaining centers and bends.)

We have now introduced $q$ in two different settings, one as a correcting multiplicative factor looking at $\langle a, b, c\rangle$, the other as a scaling factor in the $x$ direction. The following two results show that these two are connected.

Lemma 2 Given a realization of a packing with circles $\left(\sqrt{q} x_{i}, y_{i} ; b_{i}\right)$ and (possibly) $y=\sqrt{q} m_{i} x+b_{i}$, let $\mathbb{F}$ be a field containing all values $x_{i}, y_{i}, b_{i}, m_{i}$, and $q$. Then in the packing every set of three mutually tangent circles with bends $a, b, c$ satisfies $\langle a, b, c\rangle=q m^{2}$ for some $m$ in $\mathbb{F}$.

Proof We show that every triple of tangent circles with bends $b_{1}, b_{2}, b_{3}$ has $\left\langle b_{1}, b_{2}, b_{3}\right\rangle=q m^{2}$ where $m$ is in $\mathbb{F}$. First let us consider the case where all three have nonzero bends. This is illustrated in Fig. 4.

The tangency relationships give us the following three equations:

$$
\begin{aligned}
& q\left(x_{1}-x_{2}\right)^{2}+\left(y_{1}-y_{2}\right)^{2}=\left(\frac{1}{b_{1}}+\frac{1}{b_{2}}\right)^{2}, \\
& q\left(x_{1}-x_{3}\right)^{2}+\left(y_{1}-y_{3}\right)^{2}=\left(\frac{1}{b_{1}}+\frac{1}{b_{3}}\right)^{2}, \\
& q\left(x_{2}-x_{3}\right)^{2}+\left(y_{2}-y_{3}\right)^{2}=\left(\frac{1}{b_{2}}+\frac{1}{b_{3}}\right)^{2} .
\end{aligned}
$$


Fig. 4 Three circles with nonzero bends

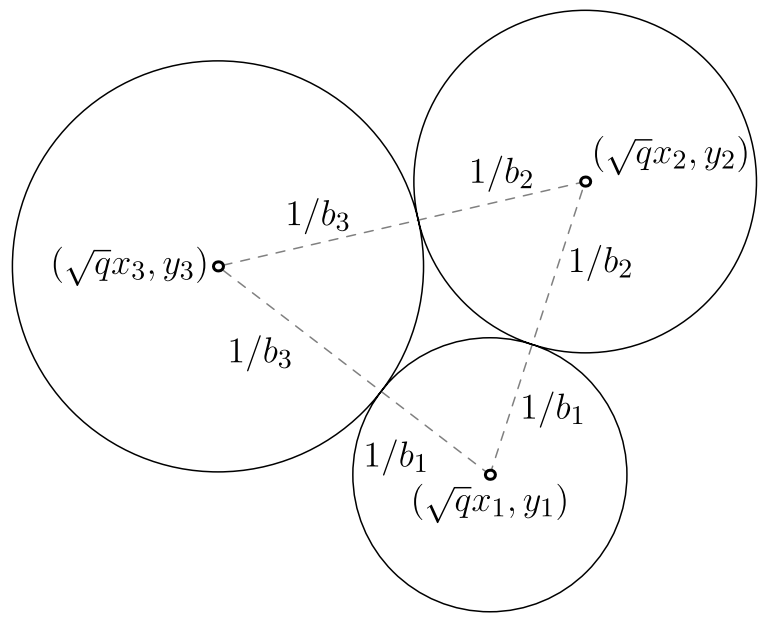

Since

$$
\begin{aligned}
\frac{16\left(b_{1} b_{2}+b_{1} b_{3}+b_{2} b_{3}\right)}{b_{1}^{2} b_{2}^{2} b_{3}^{2}}= & 4\left(\frac{1}{b_{1}}+\frac{1}{b_{2}}\right)^{2}\left(\frac{1}{b_{1}}+\frac{1}{b_{3}}\right)^{2}-\left(\left(\frac{1}{b_{1}}+\frac{1}{b_{2}}\right)^{2}\right. \\
& \left.+\left(\frac{1}{b_{1}}+\frac{1}{b_{2}}\right)^{2}-\left(\frac{1}{b_{2}}+\frac{1}{b_{3}}\right)^{2}\right)^{2}
\end{aligned}
$$

by substituting on the right-hand side using the above three equations (along with simplifying) we have

$$
\frac{16\left(b_{1} b_{2}+b_{1} b_{3}+b_{2} b_{3}\right)}{b_{1}^{2} b_{2}^{2} b_{3}^{2}}=4 q\left(-x_{1} y_{3}-y_{2} x_{3}+y_{3} x_{2}+x_{1} y_{2}+y_{1} x_{3}-y_{1} x_{2}\right)^{2},
$$

or

$$
b_{1} b_{2}+b_{1} b_{3}+b_{2} b_{3}=q\left(\frac{b_{1} b_{2} b_{3}\left(-x_{1} y_{3}-y_{2} x_{3}+y_{3} x_{2}+x_{1} y_{2}+y_{1} x_{3}-y_{1} x_{2}\right)}{2}\right)^{2} .
$$

Now suppose that one of the circles has bend 0 . Let us assume that the circles with nonzero bends are $\left(\sqrt{q} x_{1}, y_{1} ; b_{1}\right)$ and $\left(\sqrt{q} x_{2}, y_{2} ; b_{2}\right)$ with $b_{1} \geq b_{2}$. We do two transformations. First we translate

$$
(\sqrt{q} x, y) \rightarrow\left(\sqrt{q}\left(x-x_{1}\right), y-y_{1}\right) .
$$

Clearly, this does not change the field $\mathbb{F}$ needed to represent the packing. So our circles are now $\left(0,0 ; b_{1}\right)$ and $\left(\sqrt{q} x_{2}^{\prime}, y_{2}^{\prime} ; b_{2}\right)$. We now rotate as follows:

$$
\left(\begin{array}{c}
\sqrt{q} x \\
y
\end{array}\right) \rightarrow \frac{1}{1 / b_{1}+1 / b_{2}}\left(\begin{array}{cc}
y_{2}^{\prime} & -\sqrt{q} x_{2}^{\prime} \\
\sqrt{q} x_{2}^{\prime} & y_{2}^{\prime}
\end{array}\right)\left(\begin{array}{c}
\sqrt{q} x \\
y
\end{array}\right) .
$$

Since $q\left(x_{2}^{\prime}\right)^{2}+\left(y_{2}^{\prime}\right)^{2}=\left(1 / b_{1}+1 / b_{2}\right)^{2}$ (i.e., the square of the distance between the two tangent circles), this is a rotation matrix, and further it is easy to check that 
this takes $(\sqrt{q} x, y) \rightarrow\left(\sqrt{q} x^{\prime}, y^{\prime}\right)$ so that we again do not need to change the field $\mathbb{F}$. Note that this sends $\left(\sqrt{q} x_{2}^{\prime}, y_{2}^{\prime}\right) \rightarrow\left(0,1 / b_{1}+1 / b_{2}\right)$.

So applying the two transformations, we may assume that the two circles with nonzero bends are of the form $\left(0,0 ; b_{1}\right)$ and $\left(0,1 / b_{1}+1 / b_{2} ; b_{2}\right)$. If the line representing the circle with bend 0 is vertical, then it must be the case that $b_{1}=b_{2}$ and also that $1 / b_{1}= \pm \sqrt{q} a$ (this last part coming from the tangency relationship). Rearranging these, we have

$$
\left\langle b_{1}, b_{2}, 0\right\rangle=b_{1} b_{2}=b_{1}^{2}=q\left(\frac{1}{q a}\right)^{2} .
$$

Otherwise, the circle with 0 bend is of the form $y=\sqrt{q} m^{\prime} x+b^{\prime}$. We can find the slope of the line tangent to the two circles with nonzero bend which must be equal to $\sqrt{q} m^{\prime}$. Doing this, we have

$$
\pm \frac{2 \sqrt{b_{1} b_{2}}}{b_{1}-b_{2}}=\sqrt{q} m^{\prime}
$$

or, rearranging,

$$
\left\langle b_{1}, b_{2}, 0\right\rangle=b_{1} b_{2}=q\left(\frac{m^{\prime}\left(b_{1}-b_{2}\right)}{2}\right)^{2} .
$$

Finally, if two of the circles have bend 0 , then trivially we have $\left\langle b_{1}, b_{2}, b_{3}\right\rangle=0=$ $\sqrt{q} 0^{2}$.

This shows that if our centers are of the correct form, then we have a $q$-packing. In the other direction we want to show that if every set of three mutually tangent circles satisfies $\langle a, b, c\rangle=q m^{2}$ for $a, b, c, q, m$ all in $\mathbb{F}$ (i.e., we have a $q$-packing over the field $\mathbb{F}$ ), then we can position the center of the circles so that they are of the form $(\sqrt{q} x, y)$. This is a consequence of the following result.

Lemma 3 Suppose that the bends of three mutually tangent circles satisfy $\langle a, b, c\rangle=$ $q m^{2}$ for $a, b, c, m, q$ in the field $\mathbb{F}$. Further suppose that the circle of bend a is centered at $\left(\sqrt{q} x_{a}, y_{a}\right)$ (or has the form $\left.y=\sqrt{q} m_{a} x+b_{a}\right)$ and the circle of bend $b$ is centered at $\left(\sqrt{q} x_{b}, y_{b}\right)$ (or has the form $\left.y=\sqrt{q} m_{b} x+b_{b}\right)$ with all elements in $\mathbb{F}$. Then the circle of bend $c$ is centered at $\left(\sqrt{q} x_{c}, y_{c}\right)$ (or has the form $y=\sqrt{q} m_{c} x+b_{c}$ ) with all elements in the field $\mathbb{F}$.

Proof Let us first consider the case where $a$ and $b$ are nonzero. As in the proof of the previous lemma, we may first translate, rotate, and translate again while staying in the same field $\mathbb{F}$. So we will assume that $\left(\sqrt{q} x_{a}, y_{a}\right)=(0,-1 / a)$ and $\left(\sqrt{q} x_{b}, y_{b}\right)=$ $(0,1 / b)$.

Suppose that $c \neq 0$ and let $\left(x_{c}, y_{c}\right)$ be the corresponding center of the circle. We now have the following three relationships:

$$
a b+a c+b c=q m^{2},
$$




$$
\begin{aligned}
& x_{c}^{2}+\left(y_{c}+\frac{1}{a}\right)^{2}=\left(\frac{1}{a}+\frac{1}{c}\right)^{2}, \\
& x_{c}^{2}+\left(y_{c}-\frac{1}{b}\right)^{2}=\left(\frac{1}{b}+\frac{1}{c}\right)^{2} .
\end{aligned}
$$

The first of these is our assumption, while the other two come from looking at the distance between the centers of the circles.

Taking the difference of (2) and (3) and simplifying, we have

$$
y_{c}=\frac{b-a}{c(a+b)} .
$$

Substituting this into (2) and using (1), we have

$x_{c}= \pm \sqrt{\left(\frac{1}{a}+\frac{1}{c}\right)^{2}-\left(\frac{b-a}{c(a+b)}+\frac{1}{a}\right)^{2}}= \pm \sqrt{\frac{4(a b+a c+b c)}{c^{2}(a+b)^{2}}}= \pm \frac{2 m}{c(a+b)} \sqrt{q}$.

Now suppose that $c$ is 0 . If $a=b$, then we can assume that $q=1$ and we can let $x=a$ be the circle with bend 0 . Otherwise we solve for the line $y=\sqrt{q} m_{c} x+b_{c}$ using the following two equations:

$$
\begin{aligned}
& q m^{2}+1=a^{2}\left(-\frac{1}{a}-b_{c}\right)^{2}, \\
& q m^{2}+1=b^{2}\left(\frac{1}{b}-b_{c}\right)^{2},
\end{aligned}
$$

which gives the line

$$
y= \pm \frac{2 \sqrt{a b}}{a b} x+\frac{2}{b-a}= \pm \sqrt{q} \frac{2 m}{a b} x+\frac{2}{b-a} .
$$

Suppose that $a \neq 0$ and $b=0$. Then the point of intersection between the circle and line is at

$$
\left(\sqrt{q} \frac{y_{a} m_{b}+x_{a}-m_{b} b_{b}}{q m_{b}^{2}+1}, \frac{q m_{b} x_{a}+q m_{b}^{2} y_{a}+b_{b}}{q m_{b}^{2}+1}\right)=(\sqrt{q} s, t)
$$

for $s, t \in \mathbb{F}$. We can now translate this point to $(0,0)$ and then rotate so that the center of the circle with bend $a$ is located at $(0,1 / a)$ and the line becomes $y=0$ (all while staying in $\mathbb{F}$ ). If $c=0$, then use the line $y=2 / a$. If $c \neq 0$, then we have the two equations $y_{c}=1 / c$ and $q x_{c}^{2}+\left(y_{c}-1 / a\right)^{2}=(1 / c+1 / a)^{2}$. Solving, we can put the center of the circle with bend $c$ at

$$
\left(\frac{2}{\sqrt{a c}}, \frac{1}{c}\right)=\left(\sqrt{q} \frac{2}{q m}, \frac{1}{c}\right) .
$$

Finally if $a=0$ and $b=0$, then we can position $c$ at $\left(0,\left(b_{a}+b_{b}\right) / 2\right)$. 
We now use Lemma 3 to show how to place a $q$-packing into the plane. First we take two tangent circles with nonzero bends $b_{1}$ and $b_{2}$ and place their centers at $\left(0,1 / b_{1}\right)$ and $\left(0,-1 / b_{2}\right)$. These centers are in the field $\mathbb{F}$ and of the correct form. We now place circles one at a time tangent to two already present. By repeated application of the lemma all the circles will be representable in $\mathbb{F}$.

Comparing the two previous lemmas, we see that Lemma 2 shows that if all of $q, x_{i}, y_{i}, b_{i}$ are in the field $\mathbb{F}$, then so is $m_{i, j, k}=\sqrt{\left\langle b_{i}, b_{j}, b_{k}\right\rangle / q}$, while Lemma 3 can show that if all of $q, b_{i}, m_{i, j, k}$ are in the field $\mathbb{F}$, then we can assume that the $x_{i}, y_{i}$ are also in the field. Note that in the latter case the field can be determined by finding a small field (ideally smallest) containing all of $q, b_{i}, m_{i, j, k}$.

Different realizations of packings of an Apollonian configuration might lie in different fields. We would like to find a realization that uses a small field. We have already seen that (restricted) translation, dilation, and rotation will allow us to stay in the same field. The next lemma shows that we can also invert.

Lemma 4 Suppose that the bends of three mutually tangent circles satisfy $\langle a, b, c\rangle=$ $q m^{2}$ for $a, b, c, m, q$ in the field $\mathbb{F}$. We may assume (via Lemma 3 ) that the centers of the circles are of the form $\left(\sqrt{q} x_{i}, y_{i}\right)$, or that the circles are of the form $y=$ $\sqrt{q} m_{i} x+b_{i}$ with the $x_{i}, y_{i}, m_{i}, b_{i}$ in $\mathbb{F}$ for $i=a, b, c$. If we invert using a circle centered at $(\sqrt{q} v, w)$ of radius $R$ (with $v, w, R^{2}$ in $\mathbb{F}$ ), then the bends $a^{\prime}, b^{\prime}, c^{\prime}$ of the new circles are also in $\mathbb{F}$ and further satisfy $\left\langle a^{\prime}, b^{\prime}, c^{\prime}\right\rangle=q\left(m^{\prime}\right)^{2}$ for some $m^{\prime}$ in $\mathbb{F}$.

Proof We first consider the case where $a, b, c \neq 0$. Using the proof of Lemma 3, we may first through a series of translations, rotation, and (possible) reflection, assume that our circles are positioned as follows:

$$
\begin{aligned}
& \left(\sqrt{q} x_{a}, y_{a}\right)=(0,-1 / a) ; \\
& \left(\sqrt{q} x_{b}, y_{b}\right)=(0,1 / b) ; \quad \text { and } \\
& \left(\sqrt{q} x_{b}, y_{c}\right)=\left(\frac{2 m}{c(a+b)} \sqrt{q}, \frac{b-a}{c(a+b)}\right) .
\end{aligned}
$$

If we invert through a circle centered at $(\sqrt{q} v, w)$ of radius $R$, then the new bends can be expressed as follows (see [9]):

$$
\begin{aligned}
& a^{\prime}=\frac{q v^{2}+(w+1 / a)^{2}-(1 / a)^{2}}{R^{2} / a}, \\
& b^{\prime}=\frac{q v^{2}+(w-1 / b)^{2}-(1 / b)^{2}}{R^{2} / b}, \\
& c^{\prime}=\frac{q\left(v-\frac{2 m}{c(a+b)}\right)^{2}+\left(w-\frac{b-a}{c(a+b)}\right)^{2}-(1 / c)^{2}}{R^{2} / c} .
\end{aligned}
$$

From our assumptions we have that $a^{\prime}, b^{\prime}, c^{\prime}$ are in $\mathbb{F}$. Now substituting these, we have (with a little bit of simplifying)

$$
a^{\prime} b^{\prime}+a^{\prime} c^{\prime}+b^{\prime} c^{\prime}=\frac{q\left(m^{2} w^{4}-4 m v w^{2}+2 q m^{2} v^{2} w^{2}+4 v^{2}-4 q m v^{3}+q^{2} m^{2} v^{4}\right)}{R^{4}}
$$




$$
=q\left(\frac{m w^{2}+q m v^{2}-2 v}{R^{2}}\right)^{2} .
$$

Next suppose that $a, b \neq 0$ and $c=0$. Then, as in Lemma 3, we can translate so that we have $(0,1 / a ; a),(\sqrt{q}(2 / q m), 1 / b ; b)$, and $y=0$. If we invert through a circle centered at $(\sqrt{q} v, w)$ of radius $R$, then the new bends can be expressed as follows (see [9]):

$$
\begin{aligned}
& a^{\prime}=\frac{q v^{2}+(w-1 / a)^{2}-(1 / a)^{2}}{R^{2} / a}, \\
& b^{\prime}=\frac{q(v-2 / q m)^{2}+(w-1 / b)^{2}-(1 / b)^{2}}{R^{2} / b}, \\
& c^{\prime}=\frac{2 w}{R^{2}} .
\end{aligned}
$$

From our assumptions we have that $a^{\prime}, b^{\prime}, c^{\prime}$ are in $\mathbb{F}$. Now substituting these, we have, as in the previous case,

$$
\begin{aligned}
a^{\prime} b^{\prime}+a^{\prime} c^{\prime}+b^{\prime} c^{\prime} & =\frac{q\left(m^{2} w^{4}-4 m v w^{2}+2 q m^{2} v^{2} w^{2}+4 v^{2}-4 q m v^{3}+q^{2} m^{2} v^{4}\right)}{R^{4}} \\
& =q\left(\frac{m w^{2}+q m v^{2}-2 v}{R^{2}}\right)^{2} .
\end{aligned}
$$

Finally suppose that $a \neq 0$ and $b, c=0$. Then, as in Lemma 3, we can translate so that we have $(0,1 / a ; a), y=0$, and $y=2 / a$. We now have that if we invert through a circle centered at $(\sqrt{q} v, w)$ of radius $R$, then the new bends can be expressed as follows (see [9]):

$$
\begin{aligned}
a^{\prime} & =\frac{q v^{2}+(w-1 / a)^{2}-(1 / a)^{2}}{R^{2} / a}, \\
b^{\prime} & =\frac{2 w}{R^{2}}, \\
c^{\prime} & =\frac{2(2 / a-w)}{R^{2}} .
\end{aligned}
$$

From our assumptions we have that $a^{\prime}, b^{\prime}, c^{\prime}$ are in $\mathbb{F}$. Now substituting these, we have

$$
a^{\prime} b^{\prime}+a^{\prime} c^{\prime}+b^{\prime} c^{\prime}=\frac{4 q v^{2}}{R^{4}}=q\left(\frac{2 v}{R^{2}}\right)^{2} .
$$

So if all of the $q, b_{i}, m_{i, j, k}$ are in the field $\mathbb{F}$, then by inverting around a circle of an appropriate form then we still have that $q, b_{i}^{\prime}, m_{i, j, k}^{\prime}$ are also in $\mathbb{F}$. In particular we can start with some packing and place it so that a point of tangency is at the origin and all of the circles have centers of the form $(\sqrt{q} x, y)$, with the centers of the tangent circles on the $y$-axis. Now invert around a circle at the origin of radius one, and finally 


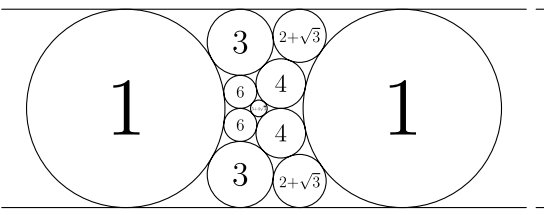

(a)

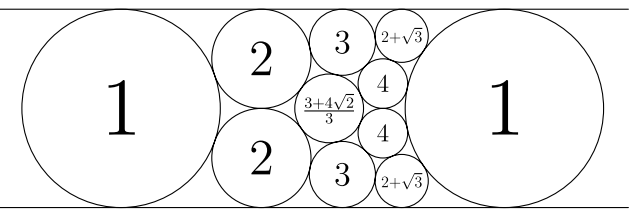

(b)

Fig. 5 Bends alone cannot determine the minimal field $\mathbb{F}$

scale and translate so that we have the three circles $(0,0 ; 1), y=1$ and $y=-1$. By the proofs of the preceding lemma all these operations can be done inside the field. If we started in a field $\mathbb{F}$, then after doing these operations we are still in the same field, although we might be able to now use a subfield in the realization. We sum this up in the following theorem.

Theorem 2 A packing is realizable as a q-packing in the field $\mathbb{F}$ if and only if any of its standard packings can be realized as a q-packing in $\mathbb{F}$.

This shows that when we are looking for a small field, we can restrict ourselves to standard packings. Moreover, it does not matter which standard packing is used, a field that realized one standard packing must realize them all.

It is tempting to think that given a standard packing, we only need to look at the smallest field that contains the bends of the packing to determine $\mathbb{F}$. This is not, in general, the case since we need to consider the centers also. In Fig. 5 we give two different standard packings of the same irreducible Apollonian configuration. A standard packing given in Fig. 5a has all bends in the field $\mathbb{Q}[\sqrt{3}]$ (the smallest circle has bend $5+4 \sqrt{3}$ ), which would seem to indicate that $\mathbb{F}=\mathbb{Q}[\sqrt{3}]$ and in particular that every standard packing would have bends in $\mathbb{Q}[\sqrt{3}]$. However, a standard packing of the same configuration given in Fig. $5 b$ has a circle with bend $(3+4 \sqrt{2}) / 3 \notin \mathbb{Q}[\sqrt{3}]$. (The correspondence of circles between these two standard packings are as follows: the left circle of bend 1 in Fig. 5 a becomes the circle with bend $(3+4 \sqrt{2}) / 3$; the circles with bend 6 become the circles with bend 2; the circles with bend 3 stay the circles with bend 3 ; the circles with bend 0 become the circles with bend 4 ; the circle with bend $5+4 \sqrt{3}$ becomes the left circle with bend 1 ; the circles with bend 4 become the circles with bend 0 ; the right circle with bend 1 stays the right circle of bend 1 ; and the circles of $2+\sqrt{3}$ stay the circles with bend $2+\sqrt{3}$.)

\subsection{Eulerian Configurations}

The value $q$ is not unique, even in the minimal field, as it can vary by scaling by a square in the field. The most interesting case is where $q$ is not a square in the field (i.e., cannot be made 1 by scaling). The case where $q$ is not a square will impose some strict conditions on the underlying tangency graph of the configuration. We say an Apollonian configuration is Eulerian if the tangency graph of the configuration is Eulerian. (A graph is Eulerian if it is connected and each vertex has even degree, or equivalently there is a walk that uses each edge exactly once.) 


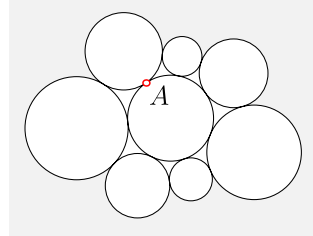

(a) Packing.

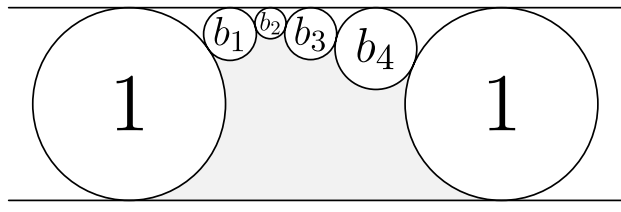

(b) Inverted.

Fig. 6 A packing where a circle is tangent to an odd number of other circles along with its inversion around point $A$

If we restrict the irreducible configurations to those which are Eulerian, this greatly reduces the number of possibilities. In particular, starting with $n=6$, there are $(1,0,1,0,2,1,5,3,18,19,79,134,501,1147, \ldots)$ Eulerian irreducible configurations (see [1]; this sequence is not yet in [10]).

Theorem 3 If a packing can be represented as a q-packing in the field $\mathbb{F}$ and $q$ is not a square in $\mathbb{F}$, then the packing is Eulerian.

Proof Suppose that there is a circle tangent to an odd number of circles; we need to show this forces $q$ to be a square. First, we illustrate the proof in the case shown in Fig. 6a. By inverting at one of the points of tangency and scaling we can get Fig. 6b, where the $b_{i}$ are in the field $\mathbb{F}$, and we have a $q$-packing.

Since this preserves $q$, we must have the following relationships (where all $\beta_{i}$ are in $\mathbb{F}): b_{1}=q \beta_{1}^{2} ; b_{1} b_{2}=q \beta_{2}^{2} ; b_{2} b_{3}=q \beta_{3}^{2} ; b_{3} b_{4}=q \beta_{4}^{2} ; b_{4}=q \beta_{5}^{2}$. Solving for the $b_{i}$, we have

$$
\begin{aligned}
& b_{1}=q \beta_{1}^{2}, \quad b_{2}=\frac{q \beta_{2}^{2}}{b_{1}}=\left(\frac{\beta_{2}}{\beta_{1}}\right)^{2}, \quad b_{3}=\frac{q \beta_{3}^{2}}{b_{2}}=q\left(\frac{\beta_{1} \beta_{3}}{\beta_{2}}\right)^{2}, \\
& b_{4}=\frac{q \beta_{4}^{2}}{b_{3}}=\left(\frac{\beta_{2} \beta_{4}}{\beta_{1} \beta_{3}}\right)^{2}=q \beta_{5}^{2} .
\end{aligned}
$$

This last relationship can only hold if $q$ is a square, concluding the proof in this case.

In general we will have after the inversion that

$$
b_{i}= \begin{cases}q \gamma_{i}^{2} & \text { if } i \text { is odd } \\ \gamma_{i}^{2} & \text { if } i \text { is even }\end{cases}
$$

where the $\gamma_{i}$ are in $\mathbb{F}$. If we started with an odd number of tangent circles, then the last $b_{i}$ must be simultaneously of the form $\gamma^{2}$ and $q \delta^{2}$, which can only be possible for $q$ a square.

\section{Constructing Subdivision Rules}

In this section we show how to form a rule on how to fill in a curvilinear triangle. We illustrate the technique with an example. Consider the rule for subdivision illustrated 


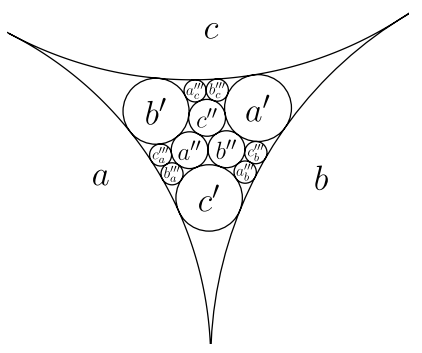

(a) General case. $b$

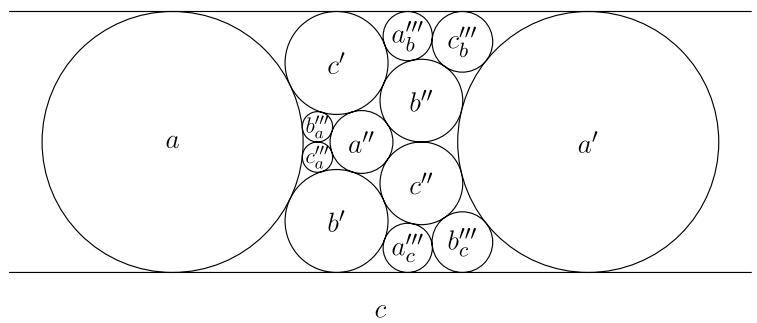

(b) Special case.

Fig. 7 A subdivision rule

in Fig. 7a, where we start with a curvilinear triangle bordered with circles having bends $a, b, c$.

We want to find a rule for finding the values of the bends of the new circles given the bends of the circles $a, b, c$. The first thing to do is to consider the special case $a=1$ and $b, c=0$, i.e., as a standard packing; this is illustrated in Fig. 7b. In this special case we find that this is a packing over $\mathbb{F}=\mathbb{Q}[\sqrt{13}]$ with $q=4-\sqrt{13}$. The centers and bends are given in Table 1.

Once we have established the special case, the key is to relate the general case to the special case. To do this we start by shifting the point of tangency between $a$ and $b$ to $(0,0)$ and then rotate so that they are of the form $(0,-1 / a ; a)$ and $(0,1 / b ; b)$. We now invert in the unit circle centered at $(0,0)$ to get the picture in Fig. 8 (where $m$ is such that $\langle a, b, c\rangle=q m^{2}$ ). We can now use the special case in Fig. $7 \mathrm{~b}$ to fill in the rest of the packing by scaling and translating the packing. We then invert again around the unit circle at the origin and then rotate and scale to put the circles back in their original position. It remains for us to relate the new bends to the old bends. In general we have that

$$
\text { new bend }=\left(d^{2}-\frac{1}{(\text { old bend })^{2}}\right)(\text { old bend }),
$$

where $d$ is the distance of the center of the circle to the point of inversion.

So suppose that we have the circle $(\sqrt{q} x, y ; \beta)$ in the special case given above. Then we scale and translate so that we now have the circle

$$
\left(\sqrt{q}\left(\frac{a+b}{4} x+\frac{m}{2}\right), \frac{a+b}{4} y+\frac{b-a}{4} ; \frac{4 \beta}{a+b}\right) .
$$

So applying the rule above, we have that the new bend $\hat{\beta}$ satisfies

$$
\begin{aligned}
\hat{\beta} & =\left(a\left(\frac{a+b}{4} x+\frac{m}{2}\right)^{2}+\left(\frac{a+b}{4} y+\frac{b-a}{4}\right)^{2}-\frac{(a+b)^{2}}{16 \beta^{2}}\right) \frac{4 \beta}{a+b} \\
& =\frac{1}{4} \beta(a+b)\left(q x^{2}+y^{2}\right)+\beta m q x+\frac{1}{2} \beta(b-a) y+\frac{1}{4} \frac{\beta^{2}-1}{\beta}(a+b)+\beta c .
\end{aligned}
$$


Table 1 Center and bends of circles in Fig. $7 \mathrm{~b}(q=4-\sqrt{13})$

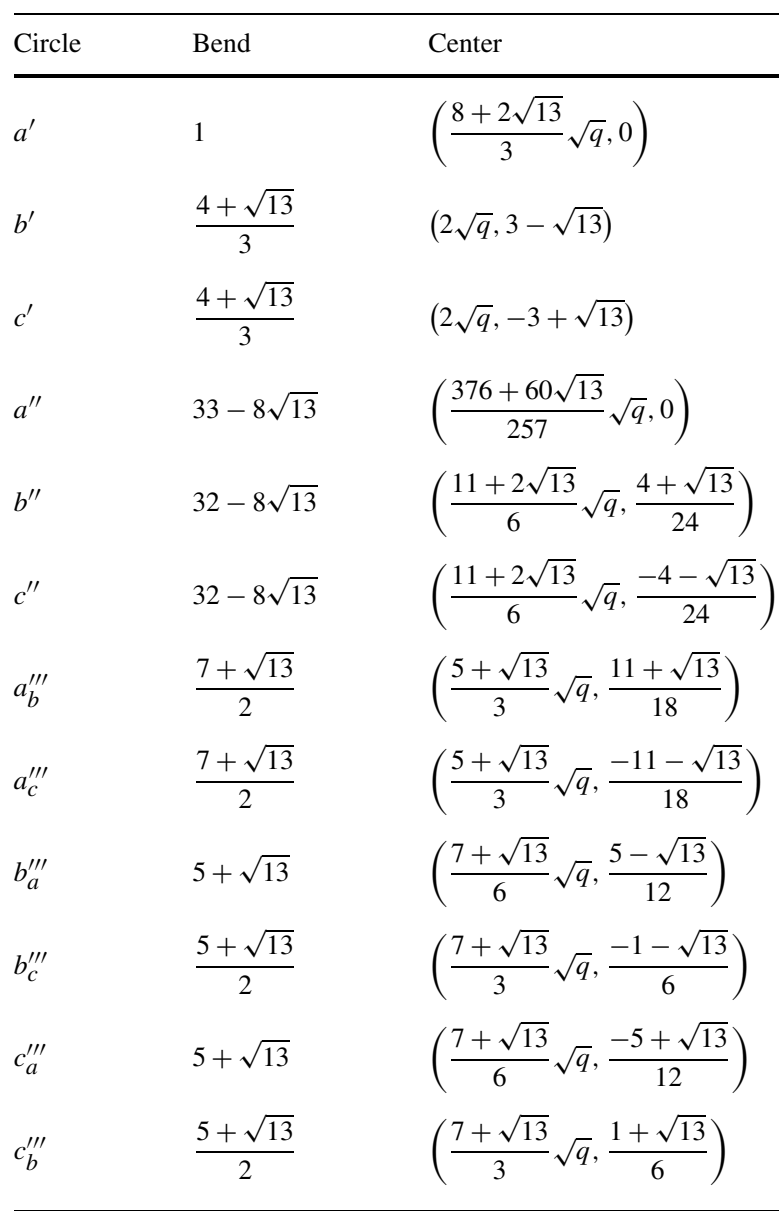

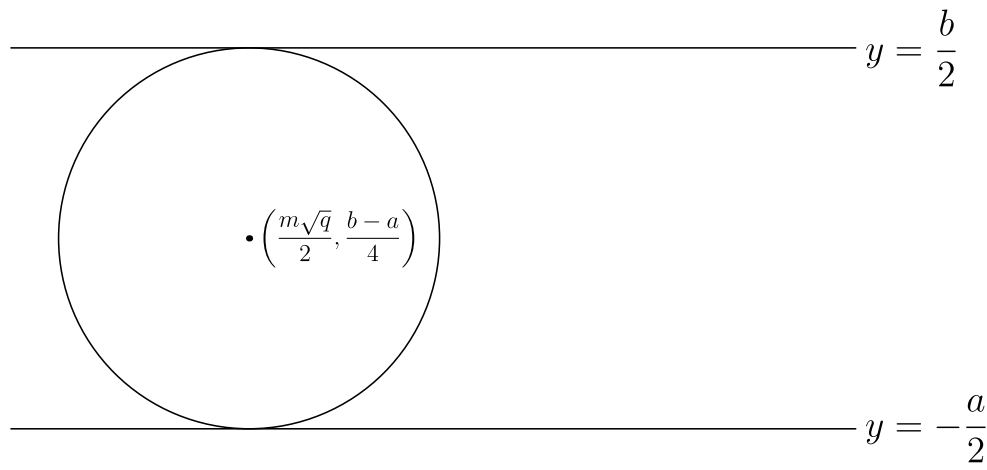

Fig. 8 The inverted figure 
This shows that the new bends are linear combinations in $\mathbb{F}$ of the quantities $a, b, c, m=\sqrt{\langle a, b, c\rangle / q}$. In particular we can apply this rule to each circle above and find a formula for the bends of the new circles in terms of the bends $a, b, c$ and quantity $m$ :

$$
\begin{aligned}
& a^{\prime}=\frac{1}{3}(4+\sqrt{13})(b+c)+2 m+a, \\
& b^{\prime}=\frac{1}{3}(4+\sqrt{13})(a+c)+2 m+b, \\
& c^{\prime}=\frac{1}{3}(4+\sqrt{13})(a+b)+2 m+c, \\
& a^{\prime \prime}=8(4-\sqrt{13})(a+b+c)+(148-40 \sqrt{13}) m+a, \\
& b^{\prime \prime}=8(4-\sqrt{13})(a+b+c)+(148-40 \sqrt{13}) m+b, \\
& c^{\prime \prime}=8(4-\sqrt{13})(a+b+c)+(148-40 \sqrt{13}) m+c, \\
& a_{b}^{\prime \prime \prime}=\frac{1}{2}(7+\sqrt{13}) a+\frac{1}{2}(5+\sqrt{13})(2 b+c)+6 m, \\
& a_{c}^{\prime \prime \prime}=\frac{1}{2}(7+\sqrt{13}) a+\frac{1}{2}(5+\sqrt{13})(b+2 c)+6 m, \\
& b_{a}^{\prime \prime \prime}=\frac{1}{2}(7+\sqrt{13}) b+\frac{1}{2}(5+\sqrt{13})(2 a+c)+6 m, \\
& b_{c}^{\prime \prime \prime}=\frac{1}{2}(7+\sqrt{13}) b+\frac{1}{2}(5+\sqrt{13})(a+2 c)+6 m, \\
& c_{a}^{\prime \prime \prime}=\frac{1}{2}(7+\sqrt{13}) c+\frac{1}{2}(5+\sqrt{13})(2 a+b)+6 m, \\
& c_{b}^{\prime \prime \prime}=\frac{1}{2}(7+\sqrt{13}) c+\frac{1}{2}(5+\sqrt{13})(a+2 b)+6 m .
\end{aligned}
$$

In general this process generalizes to any rule for filling in curvilinear triangles (we do not even need to assume irreducibility). The key to this process is (4), which shows that the bends of the new circles are linear combinations of $a, b, c, m=\sqrt{\langle a, b, c\rangle / q}$. We summarize this in the following theorem.

Theorem 4 Given a rule for filling in a curvilinear triangle, let $\mathbb{F}$ be a field containing $q, b_{i}, m_{i, j, k}$ in the case where $a=1$ and $b, c=0$. Then for any three mutually tangent circles with bends $a, b, c \in \mathbb{F}$ satisfying $\langle a, b, c\rangle=q m^{2}$ for some $m$ in $\mathbb{F}$, repeated application of the subdivision rule will have all bends of the circles in $\mathbb{F}$. Further, every three mutually tangent circles with bends $d, e, f$ will satisfy $\langle d, e, f\rangle=q m^{2}$ for some $m$ in $\mathbb{F}$.

Some of the simplest irreducible packings are shown in Fig. 9. Figures $9 \mathrm{~b}$ and $9 \mathrm{c}$ correspond to circle packings consisting of two concentric circles with respectively four and six circles in between the concentric circles. Figure 9a corresponds to the 


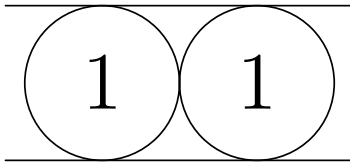

(a) $q=1$.

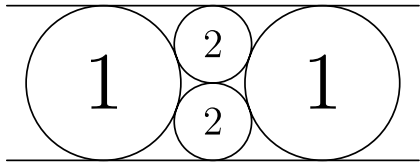

(b) $q=2$.

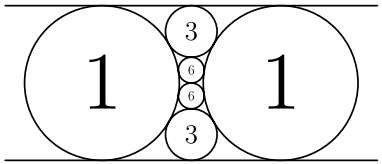

(c) $q=3$.

Fig. 9 Some small packings

traditional Apollonian case where we fill in one circle in the curvilinear triangle. The circle has $(x, y ; \beta)=(2,0 ; 1)$, so that using $(4)$, we see that in general the new bend will be $a+b+c+2 m$ (this can be used to derive Descartes Circle Theorem).

Figure $9 \mathrm{~b}$ corresponds to the packing introduced by Guettler and Mallows [8], where three circles fill in the curvilinear triangle. The circles have $(x, y ; \beta)=$ $\left(\sqrt{2}, \frac{1}{2} ; 2\right)$ and $\left(\sqrt{2},-\frac{1}{2} ; 2\right),(2 \sqrt{2}, 0 ; 1)$, so that using $(4)$, the formulas for the new bends are $a+2 b+2 c+4 m, 2 a+b+2 c+4 m$ and $2 a+2 b+c+4 m$ (note the symmetry of these equations).

Figure 9c corresponds to a packing where we fill in the curvilinear triangle using five new circles (in this case the filling is not symmetric). The circles have $(x, y ; \beta)=$ $\left(\frac{2}{3} \sqrt{3}, \frac{2}{3} ; 3\right),\left(\frac{2}{3} \sqrt{3}, \frac{1}{6} ; 6\right),\left(\frac{2}{3} \sqrt{3},-\frac{1}{6} ; 6\right),\left(\frac{2}{3} \sqrt{3},-\frac{2}{3} ; 3\right)$, and $\left(\frac{4}{3} \sqrt{3}, 0 ; 1\right)$. So the new bends are $\hat{\beta}_{1}=a+3 b+3 c+6 m, \hat{\beta}_{2}=3 a+4 b+6 c+12 m, \hat{\beta}_{3}=4 a+3 b+6 c+12 m$, $\hat{\beta}_{4}=3 a+b+3 c+6 m$, and $\hat{\beta}_{5}=\frac{4}{3}(a+b)+c+4 m$. In this case we see that we are not guaranteed that if we start with a triple of integer bends satisfying $\langle a, b, c\rangle=3 \mathrm{~m}^{2}$ for some integer $m$, then all further bends are integers. As a simple example, if we start with $a=b=c=1$, then one of the circles has bend $\frac{23}{3}$. On the other hand, if $a$ and $b$ are both divisible by 3 , then each of the resulting new circles has integer bend; further in each newly created curvilinear triangle at least two of the three circles will have bends divisible by 3 . If we start with at least two of the three circles having bend divisible by 3 , then we can always fill in each resulting curvilinear triangle so that the new bends are all integral.

As a general rule it is difficult to avoid fractions in the expressions for new bends (see (4)). However there are some packings where we are able to avoid fractions. As a good exercise, the interested reader can show if we use the (nonsymmetric) subdivision shown in Fig. 10 that if we start with three circles with bends $a, b, c \in \mathbb{Z}[\sqrt{3}]$ with $\langle a, b, c\rangle=m^{2} / 2$ for $m \in \mathbb{Z}[\sqrt{3}]$, then applying this subdivision rule arbitrarily as often as desired will still result in circles with bends in $\mathbb{Z}[\sqrt{3}]$.

In the traditional Apollonian packing, given three mutually tangent circles, we can find two circles tangent to all three, one of them filling in the interior curvilinear triangle and the other filling in the exterior curvilinear triangle. Similarly every subdivision rule can fill in both curvilinear triangles. The way that this is done is to take the packing of the special case, for example, Fig. 7b, and flip it across the $y$-axis. Now we can do the same translate, scale, and invert procedure as before; the only change to (4) is that $m$ is replaced by $-m$. 
Fig. 10 An interesting non-symmetric subdivision rule

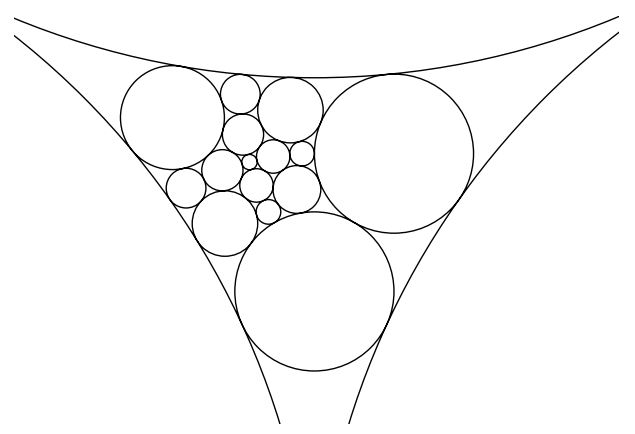

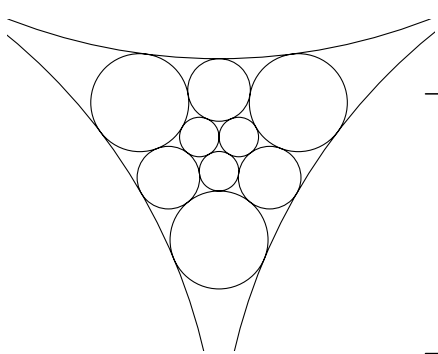

(a) Subdivision rule.

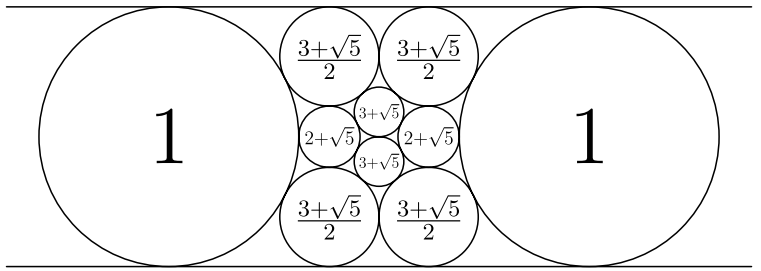

(b) Standard packing.

Fig. 11 Subdivision rule and standard configuration for icosahedron

\section{Conclusion}

We have looked at irreducible Apollonian configurations and seen how to look for a small field that can realize the configuration. We have also seen how to use these to form a rule for filling in curvilinear triangles that can then be used to construct larger configurations. Starting with an initial set of three mutually tangent circles and a set of rules, we can now apply these rules as desired. An interesting question then becomes which rules give interesting packings (either geometrically, algebraically, or combinatorially). One strong candidate for study is shown in Fig. 11. The corresponding tangency graph for this irreducible configuration is the icosahedron (the Apollonian subdivision rule, Fig. 1a, corresponds to the tetrahedron; the Guettler-Mallows subdivision rule, Fig. 1b, corresponds to the octahedron). Because of the symmetry of the icosahedron, this configuration has a unique standard packing which corresponds to $q=1$ in $\mathbb{Q}[\sqrt{5}]$.

We note that most Apollonian configurations can actually be used to create many different rules for filling in a curvilinear triangle, depending on the different standard packings. For example, the two different standard packings in Fig. 5 give rise to two different rules for filling in curvilinear triangles. In such a case we can use the same field $\mathbb{F}$ and $q$ for each rule.

Besides considering the recursive structure, we can at a more basic level look at the irreducible Apollonian configurations. We saw that for the configuration to be a $q$-packing with a nontrivial $q$, the corresponding graph of the configuration was Eulerian. It would be interesting to see what other properties of graphs translates 
Fig. 12 An extendable construction for $q=2$
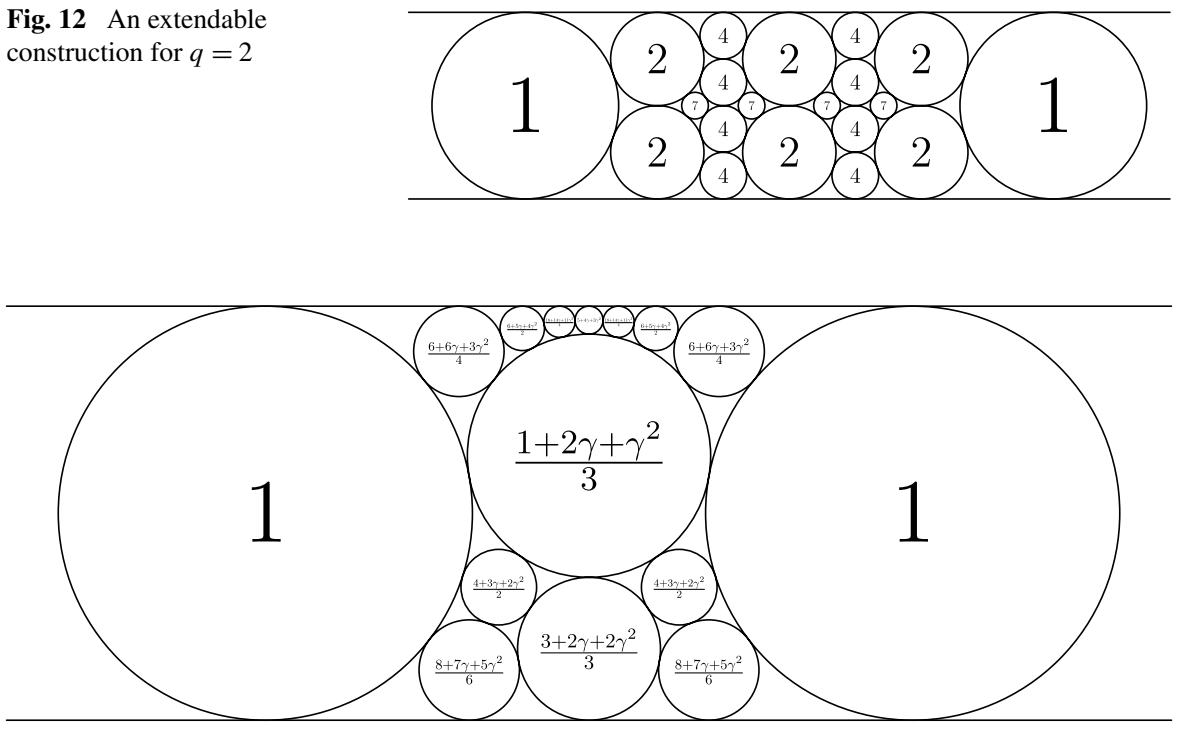

Fig. 13 A packing with $\mathbb{F}=\mathbb{Q}[\sqrt[3]{2}]($ here $\gamma=\sqrt[3]{2})$

into restrictions on the configuration and vice-versa. Many of the interesting configurations that we found exhibited symmetry in the graph; this symmetry can be used to reduce the size of the system of polynomial equations that can be solved, which might explain why configurations with small fields tend to be highly symmetric. One would expect that there is some connection between the symmetry of a graph and the minimal field; though none is currently known.

Perhaps the most interesting case is where $\mathbb{F}=\mathbb{Q}$. In this case, which values of $q$ are possible? In Fig. 9 we gave examples for $q=1,2,3$, while in Appendix we also give some additional examples for $q=6,7,21$; so far these are the only known values for $q$. It is unknown how many, if any, other values are possible. For $q=2,3$, it is known that there are infinitely many irreducible Apollonian configurations. For instance, we can extend the configuration shown in Fig. 12. A similar construction exists for $q=3$. On the other hand, for $q=6,7,21$, there are only 4, 4, 1 (respectively) known configurations.

More generally, is there any restriction on which fields are possible? Given the set of equations to be satisfied are polynomial, each irreducible configuration is realizable by some algebraic number field. We have discovered examples in the quadratic extensions $\mathbb{Q}[\sqrt{2}], \mathbb{Q}[\sqrt{3}], \mathbb{Q}[\sqrt{5}], \mathbb{Q}[\sqrt{6}], \mathbb{Q}[\sqrt{7}], \mathbb{Q}[\sqrt{13}], \mathbb{Q}[\sqrt{17}], \mathbb{Q}[\sqrt{21}]$, $\mathbb{Q}[\sqrt{37}], \mathbb{Q}[\sqrt{42}], \mathbb{Q}[\sqrt{57}], \mathbb{Q}[\sqrt{97}]$, and $\mathbb{Q}[\sqrt{105}]$. Most packings correspond to higher-order extensions; in Fig. 13 is a packing which is realizable in the cubic extension $\mathbb{Q}[\sqrt[3]{2}]$.

We have restricted our focus to Euclidean geometry. One can also look at subdivision rules in hyperbolic and spherical geometries (see [3]).

There still remain many interesting problems about Apollonian configurations. 


\section{Comments on How These Packings Were Found}

We used several techniques to find the packings discussed in this paper. Our first approach was to fix a value of $q$ and grow packings one circle at a time by hand until we got to an irreducible configuration (we limited ourselves to having rational bends). This met with some limited success, and we were able to find several packings for $q=1,2,3,6,7,21$. This method, though is quickly exhausting and discovering nontrivial examples, was, well, nontrivial.

Failing to discover new values of $q$, our next approach was to fix a standard packing (preferably one with high symmetry), then set up a system of equations relating centers and bends, and then have a computer algebra system solve them, in the hopes that we would get an interesting answer. This approach also met with some limited success; for instance, the configurations given in Figs. 7 and 11 were found using this method. This approach also had limitations in that setting up the system of equations was time consuming, and as the number of circles increased the computer had a difficult time in finding a solution.

Our last approach was to take a packing (starting with the tangency graph) and find the bends in a standard packing numerically to high precision. We could then take these numbers and try to determine algebraically what they were (checking that the algebraic answer satisfied the configuration). This approach allowed us to test many configurations quickly. Using this method, we rediscovered all of the ones we had previously found in addition to the configurations given in Figs. 10, 13, and 17 as well as many others. The Maple program used in this last approach is available from the first author's website.

Acknowledgements We express our thanks to Kenneth Stephenson for his patient explanation of the use of his CirclePack software and also for leading us to efficient algorithms to approximate and draw Apollonian configurations [2, 11]. We also express our thanks to Gunnar Brinkmann and Brendan McKay for making freely available their plantri software [1] that can quickly generate all planar triangulated graphs which correspond to irreducible configurations (as well as many other interesting planar triangulated graphs). The aid of this software made it possible to do a systematic search through the small cases revealing interesting packings. Finally, we express our thanks to the referees for their many comments which helped to improve the paper.

Open Access This article is distributed under the terms of the Creative Commons Attribution Noncommercial License which permits any noncommercial use, distribution, and reproduction in any medium, provided the original author(s) and source are credited.

\section{Appendix: Some Irreducible Configurations with $\mathbb{F}=\mathbb{Q}$}

Fig. 14 The only currently known configuration with $\mathbb{F}=\mathbb{Q}$ and $q=21$

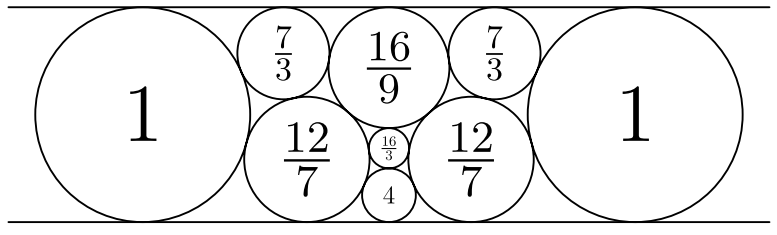




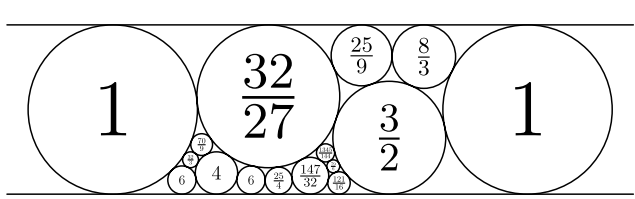

(a)

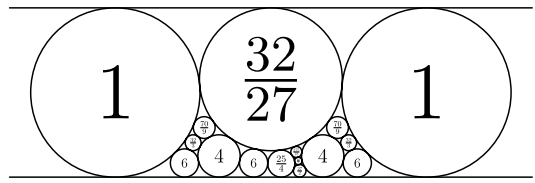

(c)

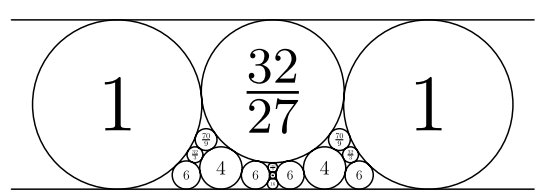

(b)

Fig. 15 The only four currently known configurations with $\mathbb{F}=\mathbb{Q}$ and $q=6$

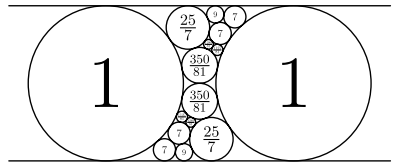

(a)

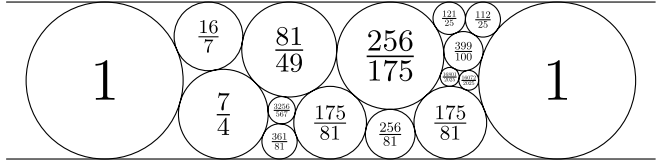

(b)

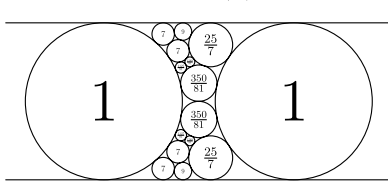

(c)

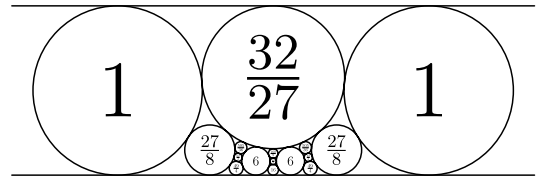

(d)

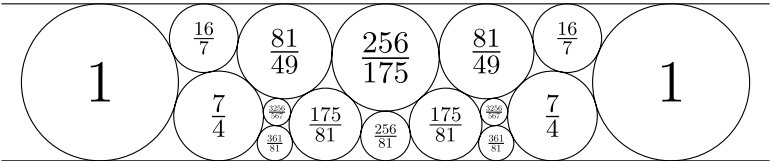

(d)

Fig. 16 The only four currently known configurations with $\mathbb{F}=\mathbb{Q}$ and $q=7$

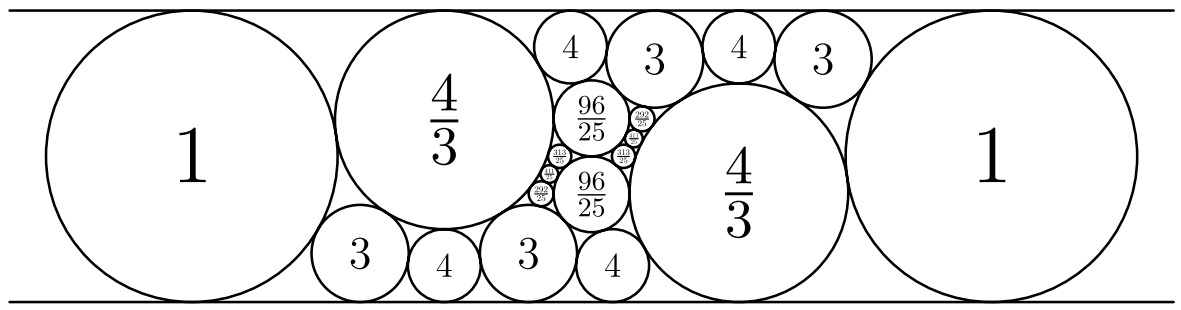

Fig. 17 Another configuration with $\mathbb{F}=\mathbb{Q}$ and $q=3$

\section{References}

1. Brinkmann, G., McKay, B.: Guide to using plantri. cs.anu.edu.au/ bdm/plantri/ (2001)

2. Collins, C., Stephenson, K.: A circle packing algorithm. Comput. Geom., Theory Appl. 25, 233-256 (2003)

3. Eriksson, N., Lagarias, J.C.: Apollonian circle packings: number theory II. Spherical and hyperbolic packings. Ramanujan J. 14, 437-469 (2007)

4. Graham, R.L., Lagarias, J.C., Mallows, C.L., Wilks, A.R., Yan, C.H.: Apollonian circle packings: number theory. J. Number Theory 100, 1-45 (2003)

5. Graham, R.L., Lagarias, J.C., Mallows, C.L., Wilks, A.R., Yan, C.H.: Apollonian circle packings: geometry and group theory I. The Apollonian group. Discrete Comput. Geom. 34, 547-585 (2005) 
6. Graham, R.L., Lagarias, J.C., Mallows, C.L., Wilks, A.R., Yan, C.H.: Apollonian circle packings: geometry and group theory II. Super-Apollonian group and integral packings. Discrete Comput. Geom. 35, 1-36 (2006)

7. Graham, R.L., Lagarias, J.C., Mallows, C.L., Wilks, A.R., Yan, C.H.: Apollonian circle packings: geometry and group theory III. Higher dimensions. Discrete Comput. Geom. 35, 37-72 (2006)

8. Guettler, G., Mallows, C.: A generalization of Apollonian packing of circles. In: Proceedings of the 20th Annual Canadian Conference on Computational Geometry, pp. 19-22 (2008)

9. Hidetoshi, F., Rothman, T.: Sacred Mathematics: Japanese Temple Geometry. Princeton University Press, Princeton (2008)

10. Sloane, N.: The On-Line Encyclopedia of Integer Sequences. www.research.att.com/ njas/sequences/ (2009)

11. Stephenson, K.: Introduction to Circle Packing. Cambridge University Press, Cambridge (2005)

12. Stephenson, K.: Circle packing: a mathematical tale. Am. Math. Soc. Not. 50, 1376-1388 (2003) 